\title{
Orientation of graphitic planes during the bias-enhanced nucleation of diamond on silicon: An x-ray absorption near-edge study
}

\author{
Ignacio Jiménez, ${ }^{\text {a) }}$ M. Mar García, and José M. Albella \\ Instituto de Ciencia de Materiales de Madrid, CSIC, 28049 Madrid, Spain \\ Louis J. Terminello \\ Lawrence Livermore National Laboratory, Livermore, California 94551
}

(Received 5 July 1998; accepted for publication 16 September 1998)

\begin{abstract}
The bias-enhanced nucleation of diamond on $\mathrm{Si}(100)$ is studied by angle-dependent $\mathrm{x}$-ray absorption near-edge spectroscopy (XANES). During diamond nucleation, a graphitic phase is also detected. The angle dependence of the XANES signal shows that the graphitic basal planes are oriented perpendicular to the surface. Implications of this result on the mechanism of bias-enhanced nucleation are discussed. (C) 1998 American Institute of Physics. [S0003-6951(98)01646-5]
\end{abstract}

The nucleation of diamond on Si during chemical vapor deposition (CVD) is greatly enhanced by application of a negative substrate bias. ${ }^{1}$ This process, often known as biasenhanced nucleation (BEN), has become very common in the growth of diamond thin films. The mechanisms leading to the nucleation enhancement are not clear, although bombardment of ions with an energy of $\sim 100 \mathrm{eV}$ seems a key condition. $^{2}$

The diamond/Si interface formed during BEN is poorly known. Some authors report an abrupt interface, ${ }^{3}$ while others propose an extended interface containing either an intermediate SiC (Ref. 4) or a graphitic interlayer. ${ }^{2}$ Formation of an abrupt $\mathrm{C} / \mathrm{Si}$ interface is somehow striking due to the large lattice mismatch between $\mathrm{C}$ and $\mathrm{Si}$ (more than 30\%). In a previous $\mathrm{x}$-ray absorption near-edge spectroscopy (XANES) study we reported the presence of graphitic phases during the bias enhanced nucleation, without $\mathrm{SiC}$ being detected. ${ }^{5}$

Structural models have proposed the presence of a graphitic phase at the interface accommodating the lattice mismatch. ${ }^{2}$ Since graphite is mechanically anisotropic, being easily compressed perpendicular to its basal planes, it seems more favorable to have the graphitic planes oriented perpendicular to the surface. This preferential orientation has not been observed so far in diamond growth, although it is well known in the case of the isoelectronic material BN. ${ }^{6}$

Angle-dependent XANES studies take advantage of the near-linear intrinsic polarization of synchrotron radiation to elucidate $\pi$ bond orientation. ${ }^{7}$ Basically, the intensity from $\pi^{*}$ states follow a cosine-squared dependence with the angle between the light and the $\pi$ bonds, according to dipole selection rules, while the $\sigma^{*}$ states are insensitive to the angle of incidence of the light. It has proved its power in the case of BN films lacking long range order which exhibited, though, a clear orientation of the basal planes. ${ }^{8,9}$ A similar study is presented here for diamond nucleation, showing that under bias conditions leading to a large nucleation density, there is a graphitic phase with the basal planes oriented perpendicular to the surface. Voltages below and above the optimal bias voltage range of $100-200 \mathrm{~V}$ result in a smaller

${ }^{\text {a)} E l e c t r o n i c ~ m a i l: ~ i j i m e n e z @ i c m m . c s i c . e s ~}$ nucleation density and the loss of the preferential orientation. ${ }^{10}$

The diamond nuclei were formed on $\mathrm{Si}(100)$ oriented wafers, using a $2.45 \mathrm{GHz}$ ASTEX microwave (MW) plasma assisted CVD system. Nucleation was favored by application of a negative dc bias to the substrate in a mixture of $\mathrm{CH}_{4}$ in $\mathrm{H}_{2}$ at a pressure of 17 Torr, with a MW power of $450 \mathrm{~W}$ and the substrate held at $850{ }^{\circ} \mathrm{C}$. Different nucleation conditions were used, with bias voltages between 50 and $250 \mathrm{~V}$, methane contents in the gas mixture between $2 \%$ and $7 \%$, and nucleation times between 5 and $60 \mathrm{~min}$.

In contrast with most of the studies on diamond nucleation, where the BEN stage is followed by a short growth time to enlarge the nuclei, here we only used BEN conditions. In this way we avoid modifications of the graphitic layer produced during diamond nucleation. Since graphite etching by the hydrogen plasma is continuously taking place, a superficial graphite layer grown under BEN conditions may be modified by the subsequent growth process.

The XANES experiments were performed at the beamline 8.2 of the Stanford Synchrotron Radiation Laboratory (SSRL). The data were collected in the total yield mode by recording the sample current to ground, and were normalized to the signal coming from a gold-covered grid located upstream in the $x$-ray path. The sample was rotated to vary the angle $\theta$ of incidence of the light from $0^{\circ}$ (normal incidence) to $90^{\circ}$ (grazing incidence).

Since the samples were grown ex situ and exposed to air prior to the XANES measurements, the presence of carbon contaminants is an important issue. All samples were measured as-received and after annealing to $400{ }^{\circ} \mathrm{C}$ in ultrahigh vacuum conditions. The $\mathrm{C}(1 s)$ and $\mathrm{O}(1 s)$ edges were measured for every sample, showing that annealing eliminates $\mathrm{C}=\mathrm{O}$ contamination, as previously reported. ${ }^{11}$

Figure 1 shows in the top panel the C $(1 s)$ XANES of a Si substrate with $\sim 30 \%$ of the surface covered with diamond nuclei before and after annealing, and in the bottom panel the spectra from a Si substrate fully covered with diamond. The spectra before annealing are displayed with continuous line and those after annealing with dots. For samples covered with a diamond film, no signal corresponding to $\mathrm{C}$ contamination is observed since the diamond surface is very inert. 


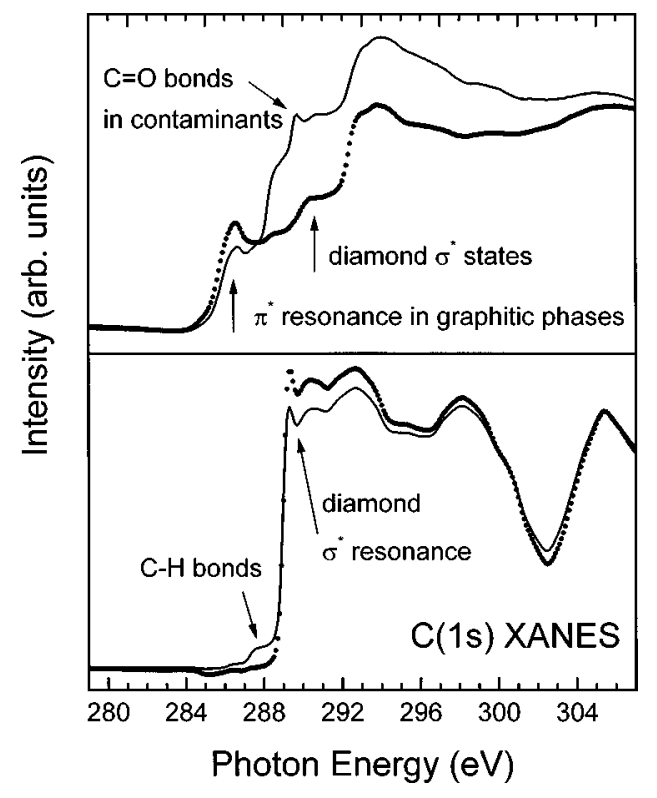

FIG. 1. Top panel: XANES spectra from a Si substrate with diamond nuclei covering $\sim 30 \%$ of the surface, asreceived (solid curve) and after annealing to $400{ }^{\circ} \mathrm{C}$ (dots). Bottom panel: spectra from a Si substrate fully covered with diamond, before and after annealing.

For samples partially covered with diamond nuclei, there is a significant contribution of carbon contamination to the $\mathrm{C}(1 s)$ spectra, that disappears upon annealing, also having its counterpart in the oxygen edge. ${ }^{11}$

We have performed angle dependent XANES measurements in several samples before and after annealing to check that the orientation effect is not an artifact produced either by carbon contaminants in the samples before annealing or by ordering of the graphitic phase after annealing. Figure 2 shows the spectra of the angle dependent measurements corresponding to a $\mathrm{Si}$ substrate with diamond nuclei covering $\sim 10 \%$ of the surface before annealing (top panel) and those from a sample with $\sim 40 \%$ of the substrate covered with diamond nuclei after annealing to $400{ }^{\circ} \mathrm{C}$ (bottom panel). BEN took place with a bias of $-200 \mathrm{~V}$. All spectra were normalized to the maximum intensity. The intensity of the $\pi^{*}$ resonance versus angle is displayed in the inset with dots, and is fitted to the equation: $I=I_{\text {ordered }} \cos ^{2} \theta+I_{\text {disordered }}$, plotted with a continuous line. The decrease of the $\pi^{*}$ intensity as the angle increases corresponds to a preferential orientation of the graphitic phase with the basal planes normal to the surface. Since the same behavior is observed in all the BEN samples before and after annealing, the intensity variation must be due to the graphitic phase formed during the BEN process, and not to any artifact from carbon contamination.

Several test measurements were also performed. To check the effect of contamination, we measured the angle dependence of the carbon signal from a Si wafer without any cleaning treatment. We did not find any dependence of the signal with the angle of incidence of the light. We also measured the angle dependence of a sample with a BEN process at a bias of $50 \mathrm{~V}$, for which no diamond nuclei were detected. The carbon signal shows no dependence with the angle either. For a BEN process with a bias of $250 \mathrm{~V}$ we find a smaller concentration of diamond nuclei than with 100 Downloaded 15 Feb 2010 to 161.111 .180 .191 . Redistribution subje

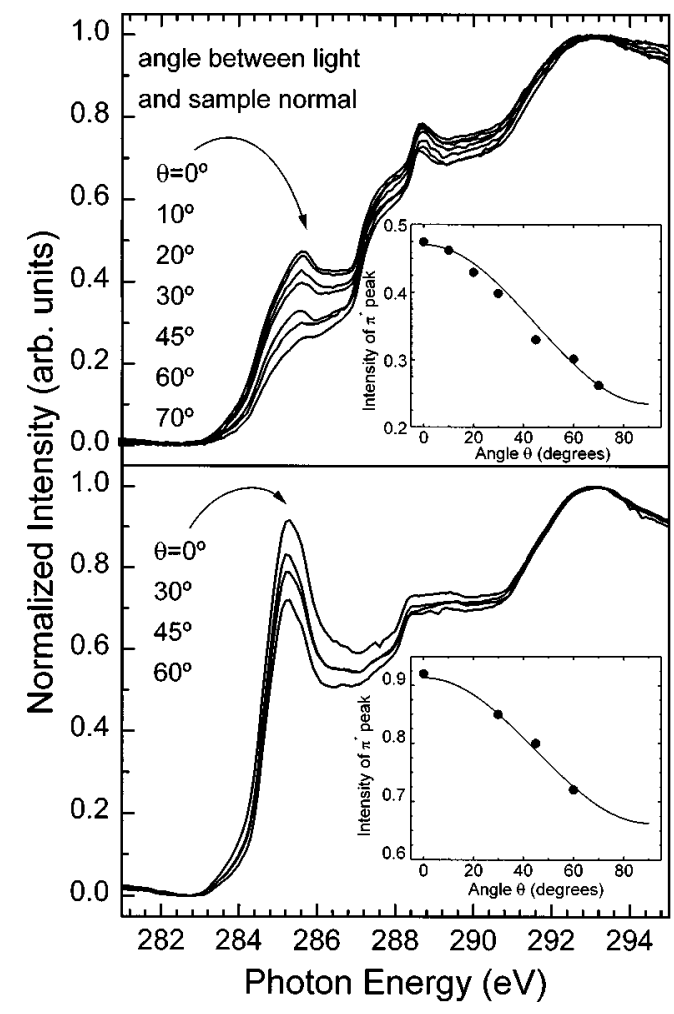

FIG. 2. Angle-dependent XANES from a BEN sample before annealing (top panel) and after annealing (bottom panel). The angle dependence of the $\pi^{*}$ resonance intensity, shown in the inset and fitted to a square-cosine law, corresponds to graphitic planes oriented normal to the surface.

$200 \mathrm{~V}$, and a graphitic intensity that changes with the angle without following any clear trend. This evidence suggests that diamond nucleation in BEN is related to the presence of oriented graphite as an interlayer between diamond and the Si substrate, and that the orientation is somehow induced by bombardment with ions of a certain energy.

Since the height of the diamond nuclei is $100-300 \AA$ (Ref. 5) and the escape depth of the electrons yielding the XANES signal is $\sim 50 \AA{ }^{12}$ we can only detect the graphitic area which is not covered by diamond nuclei. The preferential orientation of the graphitic phase appears always in connection with diamond nucleation, suggesting that it is a prerequisite for diamond formation.

The orientation of the graphitic planes can be explained by the compressive stress produced during thin film growth assisted by ion bombardment. ${ }^{13}$ Since graphite is easily compressed perpendicular to the planes, formation of an oriented graphitic layer releases the stress generated at the carbon/Si interface. The graphitic interlayer also accommodates the stress due to the lattice mismatch between diamond and $\mathrm{Si}$, enabling diamond growth. The next question is how diamond nucleation begins.

According to Robertson, the formation of $s p^{3}$ bonding can be explained by the subplantation of carbon atoms in interstitial positions beneath a graphitic surface, ${ }^{14}$ which increases locally the density of the subsurface layer, forcing the $\mathrm{C}$ atoms to adopt the denser $s p^{3}$ configuration. The existence of an optimal ion energy is explained because ions which are too slow cannot penetrate the surface layer, and ions too fast are either backscattered or produce a thermal spike that anneals the interstitial defect. This model was inito AlP license or copyright; see http://apl.aip.org/apl/copyright.jsp 
tially developed to explain the subsurface $s p^{3}$ content in amorphous carbon. In the context of diamond nucleation, the subplantantion model presents the problem of explaining the observation of diamond nuclei as surface protrusions, with a height of $100-300 \AA$, as observed by atomic force microscopy (AFM).

Based on the observation of diamond nuclei at the surface, we propose the following scenario which takes into account that the impinging carbon atoms reach the graphite planes normal to the surface. The impinging atoms can link to a single plane following the 2D layer normal to the surface, or they can bind to more than one basal plane, in which case they generate a 3D structure that forces the $s p^{3}$ hybridization. The existence of an optimal ion energy can be explained because ion bombardment facilitates the connection of basal planes by generation of defects in the hexagonal planes, but ions too energetic transform $s p^{3}$ phases into $s p^{2}$ phases. ${ }^{15}$ The final atomic arrangement according to this model is similar to that proposed by Robertson. ${ }^{2}$

At this point, it is interesting to notice some similarities between diamond and $c$-BN growth. In boron nitride, it has been reported that ion bombardment creates a high density of defects (nitrogen vacancies), ${ }^{9}$ and builds up a high biaxial compressive stress, ${ }^{16}$ that between $c$-BN and silicon there is always a layer of $h$-BN with the basal planes oriented normal to the surface, ${ }^{6}$ and that defect formation can explain the dependence of the cubic phase content with the momentum transfer in the ion collision. ${ }^{17}$ Also in $\mathrm{BN}$, when the growth is not performed under the optimal values of ion energy and substrate temperature, the cubic phase is not attained, and the orientation of the basal planes is lost. ${ }^{18}$

The similarities between diamond and $c$-BN growth suggest a related mechanism. The clue for a complete description of this mechanism may reside on the different energies involved in the growth processes for these two materials. Diamond growth is favored at substrate temperatures of $850{ }^{\circ} \mathrm{C}$ and ion energies around $100 \mathrm{eV}$, while $c$-BN growth is favored at substrate temperatures of $400{ }^{\circ} \mathrm{C}$ and ion energies around $500 \mathrm{eV}$.

In conclusion, we have found that during the BEN process, graphite oriented with the $c$ axis in the surface plane is also formed. This orientation seems related to the diamond nucleation, suggesting an extended diamond/Si interface with an oriented graphite interlayer accommodating the lattice mismatch.
This work has been partially financed by the Spanish CICYT under Projects Nos. MAT96-0529 and PB94-53, the NATO Project CRG-971539, and the U.S. Department of Energy through Lawrence Livermore National Laboratory under Contract No. W-7405-ENG-48. The work was performed at the Stanford Synchrotron Radiation Laboratory, which is supported by the DOE, Office of Basic Energy Science.

${ }^{1}$ S. Yugo, T. Kania, T. Kimura, and T. Muto, Appl. Phys. Lett. 58, 1036 (1991).

${ }^{2}$ J. Robertson, J. Gerber, S. Sattel, M. Weiler, K. Jung, and H. Ehrhardt, Appl. Phys. Lett. 66, 3287 (1995).

${ }^{3}$ L. Chang, C. J. Chen, F. R. Chen, S. F. Hu, and T. S. Lin, Diamond Relat. Mater. 5, 326 (1996).

${ }^{4}$ T.-Y. Seong, D.-G. Kim, K.-K. Choi, and Y. J. Baik, Appl. Phys. Lett. 70, 3368 (1997).

${ }^{5}$ M. M. García, I. Jiménez, L. Vázquez, C. Gómez-Aleixandre, J. M. Albella, L. J. Terminello, and F. J. Himpsel, Appl. Phys. Lett. 72, 2105 (1998).

${ }^{6}$ D. J. Kesker, D. S. Ailey, and R. F. Davis, J. Mater. Res. 8, 1214 (1993).

${ }^{7}$ R. A. Rosenberg, P. J. Love, and V. Rehn, Phys. Rev. B 33, 4034 (1986).

${ }^{8}$ A. Chaiken, L. J. Terminello, J. Wong, G. L. Doll, and C. A. Taylor II, Appl. Phys. Lett. 63, 2112 (1993).

${ }^{9}$ I. Jiménez, A. F. Jankowski, L. J. Terminello, J. A. Carlisle, D. G. J. Sutherland, G. L. Doll, W. M. Tong, D. K. Shuh, and F. J. Himpsel, Phys. Rev. B 55, 12025 (1997).

${ }^{10}$ M. M. García, I. Jiménez, L. Vazquez, J. M. Albella, and L. J. Terminello (unpublished).

${ }^{11}$ I. Jiménez, D. G. J. Sutherland, J. A. Carlisle, T. van Buuren, L. J. Terminello, and F. J. Himpsel, Phys. Rev. B 57, 13167 (1998).

${ }^{12}$ D. G. J. Sutherland, H. Akatsu, M. Copel, F. J. Himpsel, T. A. Callcot, J. A. Carlisle, D. L. Ederer, J. J. Jia, I. Jiménez, R. C. C. Perera, D. K. Shuh, L. J. Terminello, and W. M. Tong, J. Appl. Phys. 78, 6761 (1995).

${ }^{13}$ H. Windischmann, J. Appl. Phys. 62, 1800 (1987).

${ }^{14}$ J. Robertson, Diamond Relat. Mater. 2, 984 (1993).

${ }^{15}$ L. J. Huang, I. Bello, W. M. Lau, S. T. Lee, P. A. Stevens, and B. DeVries, J. Appl. Phys. 76, 7483 (1994).

${ }^{16}$ D. R. McKenzie, D. J. H. Cockayne, D. A. Muller, M. Murakawa, S. Miyake, S. Watanabe, and P. Fallon, J. Appl. Phys. 70, 3007 (1991).

${ }^{17}$ P. B. Mirkarimi, K. F. McCarty, D. L. Medlin, W. G. Wolfer, T. A. Friedmann, E. J. Klaus, G. F. Cardinale, and D. G. Howitt, J. Mater. Res. 9, 2925 (1994).

${ }^{18}$ T. A. Friedmann, K. F. McCarty, E. J. Klaus, J. C. Barbour, W. M. Clift, H. A. Johnsen, D. L. Medlin, M. J. Mills, and D. K. Ottesen, Thin Solid Films 237, 48 (1994). 